PROCEEDINGS OF THE

AMERICAN MATHEMATICAL SOCIETY

Volume 129, Number 2, Pages 505-513

S 0002-9939(00)05933-5

Article electronically published on October 12, 2000

\title{
NORMAL ESSENTIAL EIGENVALUES IN THE BOUNDARY OF THE NUMERICAL RANGE
}

\author{
NORBERTO SALINAS AND MARIA VICTORIA VELASCO \\ (Communicated by David R. Larson)
}

\begin{abstract}
A purely geometric property of a point in the boundary of the numerical range of an operator $T$ on Hilbert space is examined which implies that such a point is the value at $T$ of a multiplicative linear functional of the $C^{*}$-algebra, $C^{*}(T)$, generated by $T$ and the identity operator. Roughly speaking, such a property means that the boundary of the numerical range (of $T$ ) has infinite curvature at that point. Furthermore, it is shown that if such a point is not a sharp linear corner of the numerical range of $T$, then the multiplicative linear functional vanishes on the compact operators in $C^{*}(T)$.
\end{abstract}

In this note, we examine a purely geometric property of a point $\lambda$ in the boundary of the numerical range of a linear and bounded operator $T$, on a Hilbert space, which implies that such $\lambda$ is a normal essential eigenvalue of $T$. Roughly speaking, such a geometrical property means that the boundary curve of the numerical range has infinite curvature at that point (we must exclude however linear vertexes because they may be normal eigenvalues without being normal essential eigenvalues).

Our result allows us to give an elegant (and new) proof of a conjecture of Joel Anderson (see [1], [8, 9]):

A compact perturbation of a scalar multiple of the identity operator cannot have the closure of its numerical range equal to half a disk (neither equal to any acute circular sector).

Indeed, there are at least two corners in the boundary of such a convex set that have infinite curvature and that are not linear vertexes. So they are normal essential eigenvalues (from Corollary [2.4) which is not possible for a compact perturbation of a multiple of the identity operator.

The above mentioned result also proves, in the affirmative, a strengthening of a conjecture of Mathias Hübner [7]: such points (of infinite unilateral curvature) are in the essential spectrum of the given operator.

\section{Reducing essential eigenvalues and the numerical Range}

In this section, we recall some basic facts about the numerical range of an operator on a Hilbert space, as well as the notion of reducing essential eigenvalues and normal essential eigenvalues.

Received by the editors November 30, 1998 and, in revised form, April 29, 1999.

1991 Mathematics Subject Classification. Primary 47A12.

Key words and phrases. Infinite curvature, eigenvalue. 
Definition 1.1. Let $T$ be an operator acting on a (complex) Hilbert space $\mathcal{H}$. The numerical range and the state numerical range of $T$ are defined, respectively, as follows:

$$
\begin{gathered}
W(T):=\{\langle T x, x\rangle: x \in \mathcal{H},\|x\|=1\}, \\
W_{s}(T):=\left\{\phi T: \phi \in S\left(C^{*}(T)\right)\right\},
\end{gathered}
$$

where $S\left(C^{*}(T)\right)$ denotes the state-space of the $C^{*}$-algebra $C^{*}(T)$ generated by $T$ and the identity operator $I$ on $\mathcal{H}$.

Remark 1.2. (a) We recall [11] that $W(T)$ is always convex, and $W_{s}(T)=\overline{W(T)}$.

(b) The problem of determining the class of bounded convex sets of the form $W(T)$ for some operator $T$ on $\mathcal{H}$ is still open (an easy cardinality argument shows that there are many bounded convex sets which are not in that class). In [1] it is shown that $W(T)$ is always a Borel subset of $\mathbb{C}$, and in [9] it is proved that, when $\mathcal{H}$ is separable, the components of $W(T)$ in the boundary of $W(T)$ must be either singletons or conic arcs. On the other hand, it is not even known whether $W(T)$ can be the union of the open unit disk and a non-trivial open arc in its boundary.

Definition 1.3. Let $\lambda$ be a point in the boundary of a convex subset $C$ of the complex plane, with non-empty interior. We say that $\lambda$ is of infinite curvature if, after a suitable rotation and translation which identifies $\lambda$ with zero and puts $C$ in the upper-half plane, the real axis is a supporting line for $C$ (in which case, we shall say that $C$ is in standard position), then

$$
\lim _{\substack{\Re \alpha \rightarrow 0 \\ \Im \alpha \rightarrow 0 \\ \alpha \in C}} \frac{\Im \alpha}{\Re^{2} \alpha}=\infty .
$$

Obviously, if the boundary of $C$ is a differentiable arc at $\lambda$, then the tangent line at $\lambda$ transforms, in standard position, to the real axis and therefore we have only one choice for the standard position. Still assuming that $C$ is in standard position, we call the origin a point of left-hand infinite curvature if

$$
\lim _{\substack{\Re \alpha \rightarrow 0-\\ \Im \alpha \rightarrow 0 \\ \alpha \in C}} \frac{\Im \alpha}{\Re^{2} \alpha}=\infty .
$$

Similarly, we call the origin a point of right-hand infinite curvature if

$$
\lim _{\substack{\Re \alpha \rightarrow 0+\\ \Im \alpha \rightarrow 0 \\ \alpha \in C}} \frac{\Im \alpha}{\Re^{2} \alpha}=\infty .
$$

Remark 1.4. (a) It is clear that a suitable rotation that puts a convex set $C$ in standard position may not be unique in general, but this will not be an obstacle to study points of (unilateral or bilateral) infinite curvature. Moreover, it should be noticed that the property of having unilateral or bilateral infinite curvature depends only on the graph of the function defined by the boundary of $C$ near $\lambda=0$. Indeed, let $C$ be in a standard position. Then it is easily shown that $\lambda=0$ is of infinite curvature precisely when

$$
\lim _{\substack{\alpha \rightarrow 0 \\ \alpha \in \partial C}} \frac{\Im \alpha}{\Re^{2} \alpha}=\infty
$$


On the other hand, to have left-hand infinite curvature means that $C$ can be put in standard position so that the graph given by $\partial C$ near zero has infinite curvature on the left of zero, while to have right-hand infinite curvature means that the curvature of $\partial C$ on the right of zero is infinite.

(b) Of course, a linear vertex in the boundary of a convex set $C$ (that is a point $\lambda$ in $\partial C$ such that $C$ can be written as the convex hull of $\lambda$ and another convex set $C^{\prime}$ such that $\lambda \notin \overline{C^{\prime}}$ ) is a point of infinite curvature of $C$. In fact, it readily follows that even a corner in the boundary of a convex set is a point of infinite curvature. (We recall [8] that a point $\lambda$ in the boundary of a convex set $C$ is called a corner if $C$ is a subset of a convex set $C^{\prime}$ in which $\lambda$ is a linear vertex). But, it is easy to come up with examples of points of infinite curvature where the boundary is continuously differentiable.

(c) It is also clear that a point may be of unilateral infinite curvature but not of bilateral infinite curvature. For instance, this is the case for the origin in the convex set $C:=A \cup B$ where $A:=\{\alpha \in \mathbb{C}:-1 \leq \Re \alpha \leq 0,0 \leq \Im \alpha \leq 1\}$ and $B:=\left\{\alpha \in \mathbb{C}:(\Re \alpha)^{3 / 2} \leq \Im \alpha \leq 1,0 \leq \Re \alpha \leq 1\right\}$.

(d) The notion of unilateral infinite curvature introduced here is original. However a different formulation of the property of bilateral infinite curvature was considered in [7.

Definition 1.5. A point $\lambda \in \mathbb{C}$ is called a reducing eigenvalue of an operator $T$ if $\operatorname{Null}(T-\lambda I) \cap \operatorname{Null}\left(T^{*}-\bar{\lambda}\right) \neq\{0\}$. A point $\lambda$ is called a reducing approximate eigenvalue of an operator $T$ if there exists a sequence $u_{n}$ of unit vectors in $\mathcal{H}$ such that $\lim _{n \rightarrow \infty}\left\|(T-\lambda I) u_{n}\right\|+\left\|\left(T^{*}-\bar{\lambda} I\right) u_{n}\right\|=0$. If the sequence $u_{n}$ can be chosen so that in addition $u_{n}$ tends weakly to zero, then $\lambda$ is called a reducing essential eigenvalue. The set of all reducing approximate eigenvalues of the operator $T$ will be denoted by $R(T)$ and the set of reducing essential eigenvalues by $R_{e}(T)$.

A point $\lambda \in \mathbb{C}$ is called a normal eigenvalue of $T$ if $\lambda$ is a reducing eigenvalue of $T$ and in addition $\operatorname{Null}(T-\lambda I)=\operatorname{Null}\left(T^{*}-\bar{\lambda}\right)$. Normal eigenvalues in the Calkin algebra are called normal essential eigenvalues. More precisely (see [10]), a point $\lambda$ is called a normal essential eigenvalue of $T$ if $\lambda \in R_{e}(T)$ and, in addition, $(T-\lambda I) P$ is compact for a projection $P$ on $\mathcal{H}$ if and only if $(T-\lambda I)^{*} P$ is compact.

Remark 1.6. (a) The properties and characterizations of $R(T)$ and $R_{e}(T)$ were already discussed in [10]. In particular, $\lambda$ belongs to $R(T)$ if, and only if, there exists a $*$-homomorphism $\phi: C^{*}(T) \rightarrow \mathbb{C}$ such that $\phi T=\lambda$ and, analogously, $\lambda$ is in $R_{e}(T)$ if and only if there exists a $*$-homomorphism $\phi: C^{*}(T) \rightarrow \mathbb{C}$ such that $\phi\left(C^{*}(T) \cap \mathcal{K}\right)=0$ and $\phi T=\lambda$. (Here, we are employing the usual notation $\mathcal{K}$ for the ideal of compact operators). Thus, $R(T)$ (resp. $R_{e}(T)$ ) is contained in the intersection of $\sigma(T)$ (resp. $\sigma_{e}(T)$ ) and the complex conjugate set of $\sigma\left(T^{*}\right)$ (resp. $\left.\sigma_{e}\left(T^{*}\right)\right)$.

(b) Let $R_{00}(T)$ be the set of finite multiplicity reducing eigenvalues of $T$ which are isolated points of $R(T)$. Here, by a finite-dimensional reducing eigenvalue of $T$ we mean a complex number $\lambda$ such that $\operatorname{Null}(T-\lambda I) \cap \operatorname{Null}\left(T^{*}-\bar{\lambda} I\right)$ is a nontrivial finite dimensional subspace. Then, it is shown in [10], Theorem 6.1, that $R(T)=R_{e}(T) \cup R_{00}(T)$, where the union (of course) is disjoint.

(c) Perhaps the most important feature of reducing essential eigenvalues is the following property. Suppose that $\lambda \in R_{e}(T)$. Then, given $\varepsilon>0$, there exists a unitary operator $U_{\varepsilon}: \mathcal{H} \oplus \mathcal{H} \rightarrow \mathcal{H}$ and a compact operator $K_{\varepsilon}$ with $\left\|K_{\varepsilon}\right\|<\varepsilon$, such that $U_{\varepsilon}^{*}\left(T+K_{\varepsilon}\right) U_{\varepsilon}^{*}=T \oplus \lambda I$. 
(d) From Corollary 3.3 in [10, it follows that the points in the essential spectrum which belong to the boundary of the essential numerical range are normal essential eigenvalues. Consequently a reducing essential eigenvalue of $T$ that lies in the boundary of the numerical range $W(T)$ is automatically a normal essential eigenvalue of $T$.

\section{The MAIN RESUlT}

In this section, we prove our main result about normal essential eigenvalues in the boundary of the numerical range. We begin with a more precise and stronger version, which will be obtained starting from the next two lemmas. From now on let $T$ be an operator acting on a complex Hilbert space $\mathcal{H}$.

Lemma 2.1. If $u$ and $z$ are in $\mathcal{H}$ and $\alpha$ is a complex number, then

$$
\begin{aligned}
& |\Re[\bar{\alpha}\langle T u, z\rangle+\alpha\langle T z, u\rangle]| \\
& \quad \leq|\Re\langle T(u+\alpha z), u+\alpha z\rangle|+|\langle T u, u\rangle|+|\alpha|^{2}\|T\|\|z\|^{2} .
\end{aligned}
$$

Moreover, if $\Im\langle T v, v\rangle \geq 0$ for all $v$ in $\mathcal{H}$, then

$$
|\Im[\bar{\alpha}\langle T u, z\rangle+\alpha\langle T z, u\rangle]| \leq|\langle T u, u\rangle|+|\alpha|^{2}\|z\|^{2}\|T\| .
$$

Consequently

$$
|\Im\langle T(u+\alpha z), u+\alpha z\rangle| \leq 2\left(|\langle T u, u\rangle|+|\alpha|^{2}\|T\|\|z\|^{2}\right) .
$$

Proof. From the obvious equality

$$
\langle T(u+\alpha z), u+\alpha z\rangle=\langle T u, u\rangle+\bar{\alpha}\langle T u, z\rangle+\alpha\langle T z, u\rangle+\alpha^{2}\langle T z, z\rangle
$$

we obtain that

$$
\begin{aligned}
\mid \Re\langle T & (u+\alpha z), u+\alpha z\rangle \mid \\
& \geq|\Re[\bar{\alpha}\langle T u, z\rangle+\alpha\langle T z, u\rangle]|-|\Re\langle T u, u\rangle|-|\alpha|^{2}|\Re\langle T z, z\rangle| \\
& \geq|\Re[\bar{\alpha}\langle T u, z\rangle+\alpha\langle T z, u\rangle]|-|\langle T u, u\rangle|-|\alpha|^{2}\|T\|\|z\|^{2},
\end{aligned}
$$

which proves the first assertion of the present lemma. Now let us assume that $\Im\langle T v, v\rangle \geq 0$, for all $v$ in $\mathcal{H}$. Since $\Im\langle T(u \pm \alpha z), u \pm \alpha z\rangle \geq 0$, we conclude

$$
\begin{aligned}
& |\Im[\bar{\alpha}\langle T u, z\rangle+\alpha\langle T z, u\rangle]| \\
& \quad \leq \Im\left[\langle T u, u\rangle+|\alpha|^{2}\langle T z, z\rangle\right] \leq|\langle T u, u\rangle|+|\alpha|^{2}\|z\|^{2}\|T\| .
\end{aligned}
$$

Consequently, again from (2.1), we have,

$$
\begin{aligned}
|\Im\langle T(u+\alpha z), u+\alpha z\rangle| & \leq 2 \Im\left[\langle T u, u\rangle+|\alpha|^{2}\langle T z, z\rangle\right] \\
& \leq 2\left(|\langle T u, u\rangle|+|\alpha|^{2}\|T\|\|z\|^{2}\right) .
\end{aligned}
$$

Lemma 2.2. Given a unit vector $u$ in $\mathcal{H}$ there exist unit vectors $v$ and $w$ in $\mathcal{H}$, orthogonal to $u$ and such that $\Re\langle v, w\rangle \geq 0$, satisfying $T u=\langle T u, u\rangle u+\langle T u, v\rangle v$ and $T^{*} u=\overline{\langle T u, u\rangle} u+\overline{\langle T w, u\rangle} w$.

Proof. Put $\delta:=\langle T u, u\rangle$. From the obvious equalities $T u=\delta u+(T u-\delta u)$ and $T^{*} u=\bar{\delta} u+(T u-\bar{\delta} u)$ we easily find complex numbers $\beta$ and $\gamma$, and unit vectors $v, w$ in $\mathcal{H}$ orthogonal to $u$, such that $T u=\delta u+\beta v$ and $T^{*} u=\bar{\delta} u+\bar{\gamma} w$. By multiplying $v$ by a suitable unital complex number (and adjusting $\beta$ ), if needed, we obtain 
that $\Re\langle v, w\rangle \geq 0$. Now a straightforward calculation shows that $\beta=\langle T u, v\rangle$ and $\gamma=\langle T w, u\rangle$.

Theorem 2.3. (a) Let $0 \in \partial W_{s}(T)$ be a point of infinite (bilateral) curvature and consider $W_{s}(T)$ in standard position. If $u_{n}$ is a sequence of unit vectors in $\mathcal{H}$ such that $\left\langle T u_{n}, u_{n}\right\rangle \rightarrow 0$, then $\left\|T u_{n}\right\| \rightarrow 0$ and $\left\|T^{*} u_{n}\right\| \rightarrow 0$.

(b) Let $0 \in \partial W_{s}(T)$ be a point of right-hand (resp. left-hand) infinite curvature and consider $W_{s}(T)$ in standard position. If $u_{n}$ is a sequence of unit vectors in $\mathcal{H}$ such that $\left\langle T u_{n}, u_{n}\right\rangle \rightarrow 0$ where, for all $n,\left\langle T u_{n}, u_{n}\right\rangle$ is not zero and is contained in a segment of end points given by zero and some $\alpha_{0}$ in $W(T)$ with $\Re \alpha_{0}>0$ (resp. $\left.\Re \alpha_{0}<0\right)$, then $\left\|T u_{n}\right\| \rightarrow 0$ and $\left\|T^{*} u_{n}\right\| \rightarrow 0$.

Proof. We begin by observing that from the fact $W_{s}(T) \subset\{\lambda \in \mathbb{C}: \Im \lambda \geq 0\}$ we obtain the following elementary but important inequality:

$$
\Im\langle T v, v\rangle \geq 0, \text { for all } v \text { in } \mathcal{H} .
$$

Now we treat the case where zero is a point of infinite curvature in $\partial W_{s}(T)$, that is assertion (a). Let $u_{n}$ be a sequence of unit vectors in $\mathcal{H}$ such that $\left\langle T u_{n}, u_{n}\right\rangle \rightarrow 0$. By Lemma 2.2 we obtain unit vectors $v_{n}$ and $w_{n}$, orthogonal to $u$, such that $T u_{n}=$ $\delta_{n} u_{n}+\beta_{n} v_{n}$ and $T^{*} u_{n}=\overline{\delta_{n}} u_{n}+\bar{\gamma}_{n} w_{n}$, where $\Re\left\langle v_{n}, w_{n}\right\rangle \geq 0$, and $\beta_{n}, \gamma_{n}, \delta_{n}$, are given respectively by $\delta_{n}:=\left\langle T u_{n}, u_{n}\right\rangle, \beta_{n}:=\left\langle T u_{n}, v_{n}\right\rangle$ and $\gamma_{n}:=\left\langle T w_{n}, u_{n}\right\rangle$, for all $n$ in $\mathbb{N}$. Let $\tau_{n}$ be a complex number with $\left|\tau_{n}\right|=1$ and such that $\left|\overline{\tau_{n}} \beta_{n}+\tau_{n} \gamma_{n}\right|=$ $\left|\beta_{n}\right|+\left|\gamma_{n}\right|$. Also let $z_{n}:=v_{n}+w_{n}$, for all $n$ in $\mathbb{N}$. Since $\left\langle T u_{n}, z_{n}\right\rangle=\beta_{n}\left(1+\left\langle v_{n}, w_{n}\right\rangle\right)$ and $\left\langle T z_{n}, u_{n}\right\rangle=\gamma_{n}\left(1+\left\langle v_{n}, w_{n}\right\rangle\right)$ we have

$$
\begin{aligned}
\left|\overline{\tau_{n}}\left\langle T u_{n}, z_{n}\right\rangle+\tau_{n}\left\langle T z_{n}, u_{n}\right\rangle\right| & =\left|\overline{\tau_{n}} \beta_{n}+\tau_{n} \gamma_{n}\right|\left|1+\left\langle v_{n}, w_{n}\right\rangle\right| \\
& =\left(\left|\beta_{n}\right|+\left|\gamma_{n}\right|\right)\left|1+\left\langle v_{n}, w_{n}\right\rangle\right| .
\end{aligned}
$$

Let $r_{n}:=\left|\delta_{n}\right|^{\frac{1}{2}}$ if $\delta_{n} \neq 0$ and $r_{n}:=\frac{1}{n}$ otherwise. We define $\alpha_{n}:=r_{n} \tau_{n}$, for all $n$ in $\mathbb{N}$. From the corresponding inequality in Lemma 2.1 we obtain

$$
\left|\Im\left[\overline{\alpha_{n}}\left\langle T u_{n}, z_{n}\right\rangle+\alpha_{n}\left\langle T z_{n}, u_{n}\right\rangle\right]\right| \leq\left|\delta_{n}\right|+4 r_{n}{ }^{2}\|T\| .
$$

Since $\overline{\alpha_{n}}\left\langle T u_{n}, z_{n}\right\rangle+\alpha_{n}\left\langle T z_{n}, u_{n}\right\rangle=r_{n}\left(\overline{\tau_{n}}\left\langle T u_{n}, z_{n}\right\rangle+\tau_{n}\left\langle T z_{n}, u_{n}\right\rangle\right)$ and, by definition, $r_{n} \rightarrow 0$ and $\frac{\left|\delta_{n}\right|}{r_{n}} \rightarrow 0$, we conclude that

$$
\left|\Im\left[\overline{\tau_{n}}\left\langle T u_{n}, z_{n}\right\rangle+\tau_{n}\left\langle T z_{n}, u_{n}\right\rangle\right]\right| \rightarrow 0 .
$$

We also claim that

$$
\left|\Re\left[\overline{\tau_{n}}\left\langle T u_{n}, z_{n}\right\rangle+\tau_{n}\left\langle T z_{n}, u_{n}\right\rangle\right]\right| \rightarrow 0 .
$$

Let us define $x_{n}:=u_{n}+\alpha_{n} z_{n}$ in the case that $\Im\left[\overline{\tau_{n}}\left\langle T u_{n}, z_{n}\right\rangle+\tau_{n}\left\langle T z_{n}, u_{n}\right\rangle\right] \geq 0$ and $x_{n}:=u_{n}-\alpha_{n} z_{n}$ otherwise. The claim follows from the next two facts:

(i) Assume that $\Re\left\langle T x_{n}, x_{n}\right\rangle=0$, for all $n \in \mathbb{N}$. Then, from the first inequality in Lemma 2.1 we have that $\left|\Re\left[\overline{\alpha_{n}}\left\langle T u_{n}, z_{n}\right\rangle+\alpha_{n}\left\langle T z_{n}, u_{n}\right\rangle\right]\right| \leq\left|\delta_{n}\right|+4 r_{n}{ }^{2}\|T\|$. Consequently $\left|\Re\left[\overline{\tau_{n}}\left\langle T u_{n}, z_{n}\right\rangle+\tau_{n}\left\langle T z_{n}, u_{n}\right\rangle\right]\right| \rightarrow 0$.

(ii) Assume now that $\Re\left\langle T x_{n}, x_{n}\right\rangle \neq 0$ for all $n$ in $\mathbb{N}$. Also $\Im\left\langle T x_{n}, x_{n}\right\rangle \neq 0$ because the curvature at $\lambda=0$ is infinite (so that the only real point of $W(T)$ is zero). Furthermore, $\mu_{n}:=\frac{\Im\left\langle T x_{n}, x_{n}\right\rangle}{\Re^{2}\left\langle T x_{n}, x_{n}\right\rangle} \rightarrow \infty$, since $\left\langle T x_{n}, x_{n}\right\rangle \rightarrow 0$ with $\left\|x_{n}\right\| \rightarrow 1$. But, by the last inequality in Lemma 2.1,

$$
\left|\Re\left\langle T x_{n}, x_{n}\right\rangle\right|=\left(\frac{\Im\left\langle T x_{n}, x_{n}\right\rangle}{\mu_{n}}\right)^{\frac{1}{2}} \leq\left(\frac{2\left(\left|\delta_{n}\right|+4 r_{n}^{2}\|T\|\right)}{\mu_{n}}\right)^{\frac{1}{2}},
$$


which shows that $\frac{1}{r_{n}}\left|\Re\left\langle T x_{n}, x_{n}\right\rangle\right| \rightarrow 0$. Hence, again by Lemma 2.1 (first inequality now), $\left|\Re\left[\overline{\tau_{n}}\left\langle T u_{n}, z_{n}\right\rangle+\tau_{n}\left\langle T z_{n}, u_{n}\right\rangle\right]\right| \leq \frac{1}{r_{n}}\left|\Re\left\langle T x_{n}, x_{n}\right\rangle\right|+\frac{\left|\delta_{n}\right|}{r_{n}}+4 r_{n}\|T\|$ so that $\left|\Re\left[\overline{\tau_{n}}\left\langle T u_{n}, z_{n}\right\rangle+\tau_{n}\left\langle T z_{n}, u_{n}\right\rangle\right]\right| \rightarrow 0$.

From (i) and (ii) we conclude that (2.4) holds. By (2.3) and (2.4),

$$
\left|\overline{\tau_{n}}\left\langle T u_{n}, z_{n}\right\rangle+\tau_{n}\left\langle T z_{n}, u_{n}\right\rangle\right| \rightarrow 0
$$

By $(2.2)$, this is

$$
\left(\left|\beta_{n}\right|+\left|\gamma_{n}\right|\right)\left|1+\left\langle v_{n}, w_{n}\right\rangle\right| \rightarrow 0 .
$$

However, $\left|1+\left\langle v_{n}, w_{n}\right\rangle\right| \geq 1$ because $\Re\left\langle v_{n}, w_{n}\right\rangle \geq 0$, for all $n \in \mathbb{N}$. Thus

$$
\left|\beta_{n}\right|+\left|\gamma_{n}\right| \rightarrow 0
$$

which means precisely that $\left\|T u_{n}\right\| \rightarrow 0$ as well as $\left\|T^{*} u_{n}\right\| \rightarrow 0$. This proves (a).

In order to prove assertion (b) assume, for instance, that zero is a point of righthand infinite curvature (the case of left-hand infinite curvature can be handled similarly). Let $\alpha_{0}$ be in $W_{s}(T)$, such that $\Re \alpha_{0}>0$. Also $\Im \alpha_{0}>0$ since otherwise zero would not be a point of right-hand infinite curvature. Let $\varepsilon_{n}$ be a sequence of positive real numbers such that $\varepsilon_{n} \rightarrow 0$ and let $u_{n}$ be a sequence of unit vectors in $\mathcal{H}$ such that $\left\langle T u_{n}, u_{n}\right\rangle=\varepsilon_{n} \alpha_{0}$. As in the proof of (a), by applying Lemma 2.2, we write $T u_{n}=\delta_{n} u_{n}+\beta_{n} v_{n}$ and $T^{*} u_{n}=\bar{\delta}_{n} u_{n}+\bar{\gamma}_{n} w_{n}$, where $v_{n}, w_{n}, \delta_{n}, \beta_{n}$ and $\gamma_{n}$ are as above. However, in this case, we also have $\delta_{n}=\varepsilon_{n} \alpha_{0}$. Again, for every $n$ in $\mathbb{N}$, let $\tau_{n}$ be a complex number with $\left|\tau_{n}\right|=1$ such that $\left|\overline{\tau_{n}} \beta_{n}+\tau_{n} \gamma_{n}\right|=\left|\beta_{n}\right|+\left|\gamma_{n}\right|$, and let $z_{n}:=v_{n}+w_{n}$. As before, we obtain that

$$
\left|\overline{\tau_{n}}\left\langle T u_{n}, z_{n}\right\rangle+\tau_{n}\left\langle T z_{n}, u_{n}\right\rangle\right|=\left(\left|\beta_{n}\right|+\left|\gamma_{n}\right|\right)\left|1+\left\langle v_{n}, w_{n}\right\rangle\right| .
$$

We define $\alpha_{n}:=r_{n} \tau_{n}$, where now $r_{n}:=\left(\frac{\left|\delta_{n}\right|}{M}\right)^{\frac{1}{2}}$, for a fixed constant $M$ greater than $\frac{\left|\alpha_{0}\right|\left|\Re\left\langle T z_{n}, z_{n}\right\rangle\right|}{\Re \alpha_{0}}$ (take for example $M=\frac{5\left|\alpha_{0}\right|\|T\|}{\Re \alpha_{0}}$ ). Then,

$$
\left|\alpha_{n}\right|^{2}\left|\Re\left\langle T z_{n}, z_{n}\right\rangle\right|=\varepsilon_{n} \frac{\left|\alpha_{0}\right|\left|\Re\left\langle T z_{n}, z_{n}\right\rangle\right|}{M}<\varepsilon_{n} \Re \alpha_{0}=\Re\left\langle T u_{n}, u_{n}\right\rangle
$$

so that $\Re\left\langle T u_{n}, u_{n}\right\rangle+\left|\alpha_{n}\right|^{2} \Re\left\langle T z_{n}, z_{n}\right\rangle>0$, for all $n \in \mathbb{N}$. Hence, by defining $x_{n}:=u_{n}+\alpha_{n} z_{n}$ if $\Re\left[\overline{\tau_{n}}\left\langle T u_{n}, z_{n}\right\rangle+\tau_{n}\left\langle T z_{n}, u_{n}\right\rangle\right]>0$, and $x_{n}:=u_{n}-\alpha_{n} z_{n}$ otherwise, we obtain from (2.1) that $\Re\left\langle T x_{n}, x_{n}\right\rangle>0$. Consequently, $\Im\left\langle T x_{n}, x_{n}\right\rangle \neq 0$ for all $n \in \mathbb{N}$, since there are not positive real numbers in $W_{s}(T)$. Because zero is a point of right-hand infinite curvature and $\left\langle T x_{n}, x_{n}\right\rangle \rightarrow 0$ with $\left\|x_{n}\right\| \rightarrow 1$, it follows that $\frac{\Im\left\langle T x_{n}, x_{n}\right\rangle}{\Re^{2}\left\langle T x_{n}, x_{n}\right\rangle} \rightarrow \infty$. Then, by proceeding as in the corresponding part of the proof of (a) above (see (2.5)) we deduce that

$$
\left|\Re\left[\overline{\tau_{n}}\left\langle T u_{n}, z_{n}\right\rangle+\tau_{n}\left\langle T z_{n}, u_{n}\right\rangle\right]\right| \rightarrow 0 .
$$

Moreover, again from Lemma 2.1

$$
\left|\Im\left[\overline{\tau_{n}}\left\langle T u_{n}, z_{n}\right\rangle+\tau_{n}\left\langle T z_{n}, u_{n}\right\rangle\right]\right| \rightarrow 0
$$

(as in (2.3)). Therefore

$$
\left|\overline{\tau_{n}}\left\langle T u_{n}, z_{n}\right\rangle+\tau_{n}\left\langle T z_{n}, u_{n}\right\rangle\right|=\left(\left|\beta_{n}\right|+\left|\gamma_{n}\right|\right)\left|1+\left\langle v_{n}, w_{n}\right\rangle\right| \rightarrow 0
$$

so that $\left|\beta_{n}\right|+\left|\gamma_{n}\right| \rightarrow 0$. This proves that $\left\|T u_{n}\right\| \rightarrow 0$ and $\left\|T^{*} u_{n}\right\| \rightarrow 0$. 
Assertion (a) in the next corollary constitutes an improvement of [8], Corollary 3. Moreover assertion (b) extends [8], Corollary 4, and also shows that

every point in $\partial W(T)$ having unilateral infinite curvature but not having infinite curvature is a normal essential eigenvalue.

Assertion (c) in Corollary 2.4 improves [5], Theorem 1, and finally, assertions (a) and (c) generalize 7] (theorem and corollary).

Corollary 2.4. If $\lambda$ is a point of unilateral infinite curvature of $\partial W_{s}(T)$, then the following assertions hold:

(a) $\lambda$ is a reducing approximate eigenvalue of $T$.

(b) $\lambda$ is a normal essential eigenvalue of $T$ whenever $\lambda$ is not a linear vertex.

(c) $\lambda$ is a normal eigenvalue of $T$ whenever $\lambda$ belongs to $W(T)$.

Proof. We first observe that it is not restrictive to assume that $\lambda=0$ and that $W_{s}(T)$ is in standard position. Then, part (a) is an immediate consequence of the definition of $R(T)$ and Theorem [2.3. In order to prove (b) we first claim that a point $\lambda$ in $W(T)$ which is not a linear vertex belongs to $R_{e}(T)$. To show this claim we consider $T$ decomposed in the (direct) sum $T=A \oplus B$, acting on $H=H_{0}^{\perp} \oplus H_{0}$ where $H_{0}:=\operatorname{Ker}(T-\lambda I) \cap \operatorname{Ker}\left(T^{*}-\bar{\lambda} I\right)$. Since $A$ and $B(=0)$ are the restriction of $T$ to $H_{0}^{\perp}$ and $H_{0}$ respectively, it is clear that $\lambda$ is not a reducing eigenvalue of $A$ so, in particular, $\lambda \notin R_{00}(A)$. Notice that it is easily proved that $W(T)=$ Convhull $(\{\lambda\}, W(A))$ (see also 9$]$ ). Hence $\lambda$ must be in either $\partial W(A)$ or the complement of $W_{s}(A)$. In the first case, $W_{s}(T)=W_{s}(A)$. The second alternative is not possible since, in that case, $\lambda$ would be a linear vertex of $W_{s}(T)$ which contradicts our working assumption. Since $\lambda$ is a point of infinite curvature in $\partial W_{s}(A)$ we deduce, from (a), that $\lambda \in R(A)$. Because $\lambda \notin R_{00}(A)$, we conclude that $\lambda \in R_{e}(A)$. Consequently $\lambda \in R_{e}(T)$ and the claim is shown. Now the theorem follows from the remark made previously that a point in the boundary of $W_{s}(T)$ that is also in $R_{e}(T)$ is a normal essential eigenvalue of $T$.

To prove (c) let us assume, for instance, that $0 \in \partial W_{s}(T)$ is a point of infinite right-curvature (the case of infinite left-curvature may be handled similarly). Now assume that zero belongs to the numerical range. Then, there exists a unit vector $u$ such that $\langle T u, u\rangle=0$. Given $\alpha_{0}$ in the numerical range with $\Re \alpha_{0}>0$, there exists a unit vector $v$ such that $\langle T v, v\rangle=\alpha_{0}$. Let $x_{n}:=u+\alpha_{n} v$, for a given scalar sequence $\alpha_{n}$. Let $r_{n} \rightarrow 0$ be a sequence of positive numbers. By taking $\alpha_{n}=r_{n}$, from the condition $\Im\left\langle T x_{n}, x_{n}\right\rangle \geq 0$ we obtain

$$
\Im\langle T u, v\rangle+\Im\langle T v, u\rangle=0 .
$$

By considering now $\alpha_{n}=i r_{n}$ we deduce that $\Re\langle T u, v\rangle-\Re\langle T v, u\rangle=0$. Therefore $\langle T u, v\rangle=\overline{\langle T v, u\rangle}$. If $x=u+\alpha v$, where $\alpha$ is a given complex number, then we have $\langle T x, x\rangle=2 \Re \alpha\langle T u, v\rangle+|\alpha|^{2}\langle T v, v\rangle$. We choose $\alpha$ such that $\Re \alpha\langle T u, v\rangle=0$. Finally, let $\alpha_{n}:=s_{n} \alpha$, for a sequence of positive numbers $\left\{s_{n}\right\}$ that tends to zero and such that $\left|\alpha_{n}\right|<1$. Then $\left\langle T x_{n}, x_{n}\right\rangle=\left|\alpha_{n}\right|^{2}\langle T v, v\rangle$ which shows that $\left\langle T x_{n}, x_{n}\right\rangle$ is contained in the segment whose extreme points are $\alpha_{0}$ and 0 . Therefore, part (b) in the last theorem proves that $\left\|T x_{n}\right\| \rightarrow 0$ and $\left\|T^{*} x_{n}\right\| \rightarrow 0$. However, $x_{n} \rightarrow u$ so that $\|T u\|=\left\|T^{*} u\right\|=0$. Hence zero is a reducing eigenvalue of $T$. Also, if either $\|T u\|=0$ or $\left\|T^{*} u\right\|=0$, then $\langle T u, u\rangle=0$ so that both $\|T u\|$ and $\left\|T^{*} u\right\|$ are zero. This shows that zero is a normal eigenvalue. 
The next result proves a strengthening of a conjecture posed by Mathias Hübner in [7].

Corollary 2.5. Assume that $\lambda$ is a point of unilateral infinite curvature of $\partial W_{s}(T)$ and suppose that there is only one tangent line to $\partial W_{s}(T)$ at the point $\lambda$. Then $\lambda$ is a normal essential eigenvalue of $T$.

Proof. It follows immediately from part (b) of the previous corollary because $\lambda$ can not be a linear vertex of $\partial W_{s}(T)$.

Remark 2.6. (a) It should be pointed out that Corollary 2.4 is not valid in general if in the definition of (unilateral or bilateral) infinite curvature one replaces the power two in the denominator of the limiting expression by a higher power. This can be easily seen by considering the $2 \times 2$ matrix

$$
T=\left(\begin{array}{ll}
i & 2 \\
0 & i
\end{array}\right) .
$$

Notice that $W(T)$ is the closed disk of center $i$ and radius 1 and therefore is in standard position.

(b) It would be interesting to weaken the hypotheses of Corollary 2.4 For example, is it possible to simply assume that $\lambda$ is a point of upper-infinite curvature in $\partial W_{s}(T)$ (in the sense that we replace lim by limsup in the definition of infinite curvature)?

(c) We recall that the $m$-th matricial range of an operator $T$ on $\mathcal{H}$ is the set of $m \times m$ matrices of the form $\phi(T)$, where $\phi: C^{*}(T) \rightarrow \mathcal{M}_{m}$ is a unital completely positive linear map (see, for instance, [2]). Would it be possible to define a notion of points of infinite matricial curvature in the boundary of the matricial range of $T$, so as to be able to conclude that such points are actually in the approximate reducing matricial spectrum of $T$ (those $\phi(T)$ for which $\phi$ is actually a *-homomorphism)?

\section{JOINT REDUCING ESSENTIAL EIGENVALUES AND THE JOINT NUMERICAL RANGE}

Now, we shall find a mild extension of the last corollary to $n$-tuples.

Definition 3.1. Let $\mathbf{T}=\left(T_{1}, T_{2}, \ldots, T_{n}\right)$ be an $n$-tuple of operators acting on $\mathcal{H}$. The joint numerical range and the joint state numerical of $\mathbf{T}$ are defined, respectively, as follows:

$$
\begin{gathered}
W(\mathbf{T}):=\left\{\left(\left\langle T_{1} x, x\right\rangle,\left\langle T_{2} x, x\right\rangle, \ldots,\left\langle T_{n} x, x\right\rangle\right): x \in \mathcal{H},\|x\|=1\right\}, \\
W_{s}(\mathbf{T}):=\left\{\left(\phi T_{1}, \phi T_{2}, \ldots, \phi T_{n}\right): \phi \in S\left(C^{*}(\mathbf{T})\right)\right\},
\end{gathered}
$$

where $C^{*}(\mathbf{T})$ denotes the $C^{*}$-algebra generated by $\mathbf{T}$ and the identity operator on $\mathcal{H}$.

Remark 3.2. (a) $W_{s}(\mathbf{T})$ is always convex but, in general, $W_{s}(\mathbf{T})=\overline{W(\mathbf{T})}$ only for $n=1$. Indeed, for $n>1$ the above equality does not always hold and $W(\mathbf{T})$ is not convex in general [3].

(b) If $\lambda$ is an extreme point of $\partial W_{s}(\mathbf{T})$, for a given $n$-tuple of operators $\mathbf{T}$, then $\lambda \in \overline{W(\mathbf{T})}$. Indeed, the proof of this fact uses a standard argument: let $\Sigma_{\lambda}$ be the subset of $S\left(C^{*}(\mathbf{T})\right)$ consisting of those states $\phi$ on $C^{*}(\mathbf{T})$ such that $\phi(\mathbf{T})=\lambda$. Since $\Sigma_{\lambda}$ is compact and convex in the $w^{*}$-topology, there exists an extreme point $\psi$ in $\Sigma_{\lambda}$. Using the fact that $\lambda$ is an extreme point of $W(\mathbf{T})$, it readily follows that 
$\psi$ is a pure state of $C^{*}(\mathbf{T})$. By Glim's Lemma (see [4] and [6]), we obtain that $\psi$ is in the $w^{*}$-closure of the set of vector states of $C^{*}(\mathbf{T})$. Thus, evaluating at $\mathbf{T}$, we conclude that $\lambda \in \overline{W(\mathbf{T})}$, as desired.

(c) In the next theorem we use the notions of joint reducing approximate point spectrum and of joint reducing essential spectrum of an $n$-tuple of operators. These are the natural extensions to $n$-tuples of the corresponding notions for single operators.

Theorem 3.3. Given an $n$-tuple $\mathbf{T}=\left(T_{1}, \cdots, T_{n}\right)$ of operators acting on $\mathcal{H}$, assume that $\lambda=\left(\lambda_{1}, \cdots, \lambda_{n}\right) \in \partial W_{s}(\mathbf{T})$ satisfies $\lambda_{j}$ is a point of unilateral infinite curvature of $\partial W_{s}\left(T_{j}\right)$, for $1 \leq j \leq n$. Then $\lambda \in R(\mathbf{T})$. If, furthermore, $\lambda$ is not a linear vertex of $\partial W(\mathbf{T})$, then $\lambda$ is a joint normal essential eigenvalue for $\mathbf{T}$.

Proof. The proof consists of a repeated application of Corollary 2.4, by projecting in each coordinate. We should point out that under the present hypotheses, $\lambda$ is an extreme point of $\overline{W(\mathbf{T})}$.

\section{REFERENCES}

1. J. Agler, Geometric and topological properties of the numerical range Indiana Univ. Math. J. 31 (1982), 767-777 MR 84i:47004

2. J. Bunce \& N. Salinas, Completely positive maps on $C^{*}$-algebras and the left matricial spectra of an operator Duke Math. J. 74 (1976), 747-773 MR 55:3798

3. A. T. Dash, Joint numerical ranges, Glasnik Matematicki (1972) 75-81 MR 48:2795

4. J. Dixmier, Les $C^{*}$-algèbres at leurs represéntations Gauthier-Villars, Paris (1964) MR 30:1404

5. W. Donoghue, On the numerical range of a bounded operator Michigan Math. J. 4, (1957), 261-263 MR 20:2622

6. J. Glimm, A Stone-Weierstrass theorem for $C^{*}$-algebras Ann. Math. 72 (1960), 216-244 MR 22:7005

7. M. Hübner, Spectrum where the boundary of the numerical range is not round Rocky Mountain J. Math. 25 (1995), 1351-1355 MR 96k:47007

8. J. Lancaster, The boundary of the numerical range Proc. Amer. Math. Soc. 49 (1975), 393-398 MR 51:8851

9. M. Radjabalipour \& H. Radjavi, On the geometry of numerical ranges Pacific J. Math. 61 (1975), 507-511 MR 53:3732

10. N. Salinas, Reducing essential eigenvalues Duke. Math. J. 40 (1973), 561-580 MR 52:11639

11. J. G. Stampfli \& J.P. Willians, Growth conditions and the numerical range in a Banach algebra Tōhoku Math. Jour. 20 (1968), 417-424 MR 39:4674

Department of Mathematics, The University of Kansas, Lawrence, Kansas 66045

E-mail address: norberto@kuhub.cc.ukans.edu

Departamento de Análisis Matemático, Universidad de Granada, 18071 Granada, SPAIN

E-mail address: vvelasco@goliat.ugr.es 